This item was submitted to Loughborough's Research Repository by the author.

Items in Figshare are protected by copyright, with all rights reserved, unless otherwise indicated.

\title{
Modelling the deposition process on the CdTe/CdS interface
}

PLEASE CITE THE PUBLISHED VERSION

https://doi.org/10.1016/j.nimb.2017.09.012

PUBLISHER

(c) Elsevier

VERSION

AM (Accepted Manuscript)

PUBLISHER STATEMENT

This work is made available according to the conditions of the Creative Commons Attribution-NonCommercialNoDerivatives 4.0 International (CC BY-NC-ND 4.0) licence. Full details of this licence are available at: https://creativecommons.org/licenses/by-nc-nd/4.0/

\section{LICENCE}

CC BY-NC-ND 4.0

\section{REPOSITORY RECORD}

Yu, Miao, and Steven Kenny. 2017. "Modelling the Deposition Process on the Cdte/cds Interface". figshare. https://hdl.handle.net/2134/26552. 


\title{
Modelling the deposition process on the $\mathrm{CdTe} / \mathrm{CdS}$ interface
}

\author{
Miao YU ${ }^{\mathrm{a}}$, Steven D. KENNY ${ }^{\mathrm{b}}$ \\ ${ }^{a}$ School of Mathematics and Statistics, Xidian University, Xi'an, Shaanxi, 710071, China \\ ${ }^{b}$ Department of Materials, Loughborough University, Loughborough, Leicestershire, LE11 \\ 3TU, United Kingdom
}

\begin{abstract}
CdTe is an excellent material for low-cost, high efficiency thin film solar cells and holds the record for Watts $\$$ performance [1, 2]. Defects such as grain boundaries and dislocations lower the efficiency of CdTe solar cells [3], thus it is important to do research on how these defects are formed during the growth process, especially on the interfaces of different materials.

In this work we use computer simulation to predict the growth of a sputter deposited CdTe thin film on the CdS surfaces. Single deposition tests have been performed, to study the behaviour of deposited clusters under different conditions.

We deposit a $\mathrm{Cd}_{x} \mathrm{Te}_{y}(x, y=0,1)$ cluster onto the wurtzite (111) Cd and $\mathrm{S}$ terminated CdS surfaces with energies ranging from 1 to $40 \mathrm{eV}$. More than 1,200 simulations have been performed for each of these cases so as to sample the possible deposition positions and to collect sufficient statistics. The results show that $\mathrm{Cd}$ atoms are more readily sputtered from the surface than Te atoms and the sticking probability is higher on $\mathrm{S}$ terminated surfaces than $\mathrm{Cd}$ terminated surfaces. They also show that increasing the deposition energy typically leads to an increase in the number of deposited atoms replacing surface atoms and tends to decrease the number of atoms that sit on the surface layer, whilst increasing the number of interstitials observed.
\end{abstract}

Email addresses: MYu@idian.edu.cn (Miao YU), S.D.Kenny@1boro.ac.uk (Steven D. KENNY )

Preprint submitted to Elsevier

September 4, 2017 
Keywords: Modelling, CdTe, CdS, Energetic impact

\section{Introduction}

With the development of modern society, the world's energy demands are increasing, therefore the security and supply of energy is a key problem. Conventional non-renewable resources, such as coal, oil, natural gas and nuclear, are

5 still the main energy sources, but the reserves of these non-renewable resources are limited, and also the usage of these resources could cause many environmental problems. 4. People need to find more environmentally friendly, renewable energy, and solar power is one of the most promising renewable energies.

Electricity is one of the most common sources of energy for daily use. The majority of electricity is generated by conventional non-renewable sources. Governments are taking efforts in developing renewable electricity stations. In the UK, renewables share of electricity generation was over a quarter in 2016 Q1, up 2.3 percentage points on the share in 2015 Q1 [5] and solar photovoltaics (PVs) have an important role to play in this [6.

Solar PVs is now the third most important renewable energy source in terms of globally installed capacity. By the end of 2015, its capacity increased 29 percent year-on-year to a running total of $229 \mathrm{GW}$ worldwide, and $700 \mathrm{GW}$ of total global installed solar power is possible by 2020. China alone installed $14 \%$ of newly installed solar power sectors in 2016. 7] By far, the most prevalent material for solar cells is crystalline silicon. But thin film PVs devices have great potential and are a cheaper technology than conventional Si based photovoltaic devices $[8]$.

Cadmium Telluride (CdTe) is an excellent material for low-cost, high efficiency thin film solar cells, and it is the only thin film photovoltaic technology to surpass crystalline silicon PVs in the watt/cost measure and have promising efficiency 1, 2]. However the laboratory record efficiency of CdTe solar cells lags significantly behind the theoretical maximum for the material. This discrepancy is often attributed to defects such as grain boundaries and intra-grain 
dislocations [3]. Thus it is important to do research on how these defects are formed during the growth process, especially on the interface of two materials, and therefore reduce them.

In the laboratory, many interesting process are unable to be captured directly during an experiment due to the extremely short time and size scale. In materials science, scientists usually use atomistic simulations as an outstanding

35 partner with experiment in addressing problems. By changing the parameters in the simulations, we can simulate different experimental methods for producing thin film cells, e.g. magnetron sputtering [9] and close space sublimation [10]. We use computer simulation to gain knowledge and predict the growth of the sputter deposited thin film PVs.

40 Molecular dynamics (MD) is one of the atomistic simulation techniques used in material sciences. In this method, an appropriate interatomic potential is chosen to describe the atomic forces, and the motion of atoms can be simulated by solving Newton's equations of motion. One can model the dynamics by integrating the equations of motion numerically.

MD follows the actual dynamical evolution of the system. The technique has been able to model many interesting processes, such as sputtering [11, crack propagation [12] and nanoindentation [13]. Resolving individual atomic vibrations requires a time step of the order of femtoseconds (fs) to integrate of the equations of motion.

Early research studies the energetic impacts of individual $\mathrm{Cd}_{x} \mathrm{Te}_{y}(x, y=$ $0,1)$ clusters and the growth process on the CdTe surfaces. [14, 15] These research helps us understanding the growth of CdTe thin films. It is quite interesting to do more research on the growth process on the $\mathrm{CdTe} / \mathrm{CdS}$ interface, since these two materials have different lattice constant, and therefore more likely to form defects. In this report, we use the MD to simulate the impact of individual $\mathrm{Cd}_{x} \mathrm{Te}_{y}(x, y=0,1)$ clusters on the $\mathrm{CdS}$ surfaces. These energetic impact tests are helpful to understand how the atoms behave during the deposition process in different situations, and therefore helpful to find the appropriate growth conditions [16, 17]. 


\section{Methodology}

We use the MD to simulate the individual energetic impact tests, which generally last for a few picoseconds (ps). The MD code we are using for the simulations is the LAMMPS package (Large-scale Atomic/Molecular Massively Parallel Simulator [18, 19]), an open source code using classical MD.

To simulate the impacts on the CdTe systems, we use Stillinger-Weber (SW) potentials 20] for the II-VI elements [21]. The SW potentials are the most widely used semiconductor interatomic potentials. They use an energy penalty for non-tetrahedral bond angles to ensure the tetrahedral structure as the lowest energy structures. All II-VI compounds, including CdTe and CdS, exhibit either a zinc-blende or wurtzite structure in the stoichiometric conditions. As observed in experiments, only zinc-blende or wurtzite structure occurs during growth simulations under stoichiometric conditions, thus the SW potential may realistically reveal the defect formation mechanisms.

The lattice structure of CdS is wurtzite as shown in Figure B.1. The grey 75 spheres represent the $\mathrm{Cd}$ atoms and yellow spheres the $\mathrm{S}$ atoms. The lattice constants for the CdS in our systems are chosen to be $a=4.126 \AA$ and $c=$ $6.737 \AA$, which are the optimal lattice constants using the SW potentials. In the experiments, CdTe are deposited on the wurtzite (111) CdS surfaces. Since the wurtzite (111) surfaces have the same hexagonal structure as the zinc-blende structure, CdTe would grow in (111) or (100) directions due to the different lattice constants, which are most common types of zinc-blende type of surfaces observed in the experiments. We simulate individual $\mathrm{Cd}_{x} \mathrm{Te}_{y}(x, y=0,1)$ cluster impact simulations on two different surfaces: the Cd-terminated (111) surface and the Te-terminated (111) surface.

The side view and top view of a wurtzite (111) S-terminated CdS surface are shown in Figure B.2. Bigger yellow spheres represent $\mathrm{S}$ atoms, and smaller green spheres represent $\mathrm{Cd}$ atoms. It is clear that the lattice has a double-layer structure, and the surface atoms are in a hexagonal shape. The red triangle areas in Figure B.2b indicate the smallest repeatable regions on the surface, 
and the impact simulations are done within this region.

We model 6 double-layers of atoms, in total of 960 atoms, for both (111) Cd-terminated surface system and (111) S-terminated surface system. The bottom double-layer is fixed, and the next double-layer above the fixed zone are thermalised.

The growth conditions vary for different experimental techniques. We model the growth of CdTe thin films by magnetron sputtering deposition, which is a widely used technique for CdTe thin film production. In sputtering room temperature deposition is the most common, but elevated temperatures can be used to optimise performance. 22] Thus in our impact simulations, the temperature is set to be $350 \mathrm{~K}$. We use the Berendsen thermostat [23] to thermalise the system.

A single $\mathrm{Cd}_{x} \mathrm{Te}_{y}(x, y=0,1)$ cluster, namely a single $\mathrm{Cd}$ atom, a single $\mathrm{Te}$ atom or a single CdTe cluster, is deposited onto the lattice at the height of approximately $10 \AA$ above the surface. The atom or cluster is given a velocity perpendicular to the surface, which is equivalent to be given a deposition energy of $1 \mathrm{eV}, 10 \mathrm{eV}, 20 \mathrm{eV}$ or $40 \mathrm{eV}$. The position of the deposited cluster is chosen randomly within the smallest area of each surfaces (the red regions shown in Figure B.2b. We perform the MD simulation for 4 ps to allow the impact to fully equilibrate. We then analyse the behaviour of the deposited cluster.

We did the impact simulations of 3 different species/clusters onto 2 surfaces with 4 different impact energies respectively, in total 24 cases. For each of these cases, we performed more than 1,200 impact simulations at random positions and rotations so as to sample the possible deposition positions and to collect sufficient statistics.

\section{Impact results}

From all the impact results, we have studied the behaviour of the deposited cluster, i.e. where is the deposited cluster at the final state and how does it affect the surrounding atoms. We categorize the final states into seven cases as 
illustrated in Figure B.3 and described in Table 1 .

\begin{tabular}{|c|c|c|}
\hline Case 1 & Bounce off & The deposited cluster bounces off the surface; \\
\hline Case 2 & Sputter out & $\begin{array}{l}\text { The deposited cluster collides and other atoms are } \\
\text { ejected from the surface; }\end{array}$ \\
\hline Case 3 & Replace & $\begin{array}{l}\text { The deposited cluster replace one of the surface atoms } \\
\text { and push it onto the surface. }\end{array}$ \\
\hline Case 4 & Sit on & The deposited cluster sits on the surface as a new layer; \\
\hline Case 5 & Join first layer & $\begin{array}{l}\text { The deposited cluster joins the first layer and forms de- } \\
\text { fects in the surface layer; }\end{array}$ \\
\hline Case 6 & Penetrate & $\begin{array}{l}\text { The deposited cluster penetrates the surface and be- } \\
\text { comes interstitials; }\end{array}$ \\
\hline
\end{tabular}

Table 1: Classification of impact results.

Percentage bar charts with errors are generated for each of the 24 cases shown in Figures B.4.

The results show that on the Cd-terminated surface, increasing the deposition energy typically leads to a decrease in the number of atoms that sit on the layer. It also tends to increase the number of atoms sputtered from the system, 125 replace the surface atoms or even penetrate the surface. On the S-terminated surface, the results show a similar tend, except there is almost no penetration nor joining of the surface layer.

The behaviours of the deposited cluster at low energies are less complex than ones at higher energies as expected. The deposited clusters with higher energies could penetrate the surface and displace other atoms to other sites, while ones with lower energies usually lose their energy in the impact and stick on the surface.

The results show that, it's more difficult for the cluster to penetrate the S-terminated surfaces than the Cd-terminated ones. The Cd atoms are more 


\section{Conclusion}

The energetic impact simulations of small $\mathrm{Cd}_{x} \mathrm{Te}_{y} \quad(x, y=0,1)$ clusters onto the wurtzite (111) $\mathrm{Cd}$ and $\mathrm{S}$ terminated surfaces at 1, 10, 20 and $40 \mathrm{eV}$ have been simulated.

The results show that $\mathrm{Cd}$ atoms are more readily sputtered than $\mathrm{Te}$ and $\mathrm{S}$ atoms, especially on the Cd-terminated surfaces. The deposited clusters are more likely to be reflected than sputtered. The sticking probability is higher for the S-terminated surfaces than the Cd-terminated ones. The Cd-terminated 
surfaces more likely to be penetrated or create interstitals in the surface layer CdTe layers.

\section{Appendix A. References}

n [1] W. Shockley, H. J. Queisser, Detailed Balance Limit of Efficiency of p-n Junction Solar Cells, Journal of Applied Physics 32 (3) (1961) 510-519. doi:10.1063/1.1736034,

URL http://link . aip.org/link/JAPIAU/v32/i3/p510/s1\&Agg=doi

[2] M. A. Green, Radiative efficiency of state-of-the-art photovoltaic cells, Progress in Photovoltaics: Research and Applications 20 (4) (2011) 472476. doi:10.1002/pip.1147 URL http://doi .wiley.com/10.1002/pip.1147

[3] J. Y. W. Seto, The electrical properties of polycrystalline silicon films, Journal of Applied Physics 46 (12) (1975) 5247-5254. doi:10.1063/1. 321593

URL http://link.aip.org/link/JAPIAU/v46/i12/p5247/s1\&Agg=doi

[4] S. Shafiee, E. Topal, When will fossil fuel reserves be diminished?, Energy policy 37 (1) (2009) 181-189. doi:10.1016/j.enpol.2008.08.016.

口 URL http://linkinghub.elsevier.com/retrieve/pii/ S0301421508004126

190

[5] Department of Energy \& Climate Change, Energy Trends: June 2016, Energy Trends (2016) 1-80. 
[6] H. Spanggaard, F. C. Krebs, A brief history of the development of organic and polymeric photovoltaics, Solar Energy Materials and Solar Cells. URL http://www.sciencedirect.com/science/article/pii/ S0927024804000923

[7] Solar Power Europe, Global Market Outlook For Solar Power 2016-2020, Global Market Outlook (2017) 1-40.

[8] G. Di Marco, L. Palmisano, Thin-Film Photovoltaics, International Journal of Photoenergy 2010 (2010) 1-2. doi:10.1155/2010/528512. URL http://www .hindawi .com/journals/ijp/2010/528512/

[9] A. D. Compaan, A. Gupta, S. Lee, S. Wang, J. Drayton, High efficiency, magnetron sputtered CdS/CdTe solar cells, Solar Energy. URL http://www.sciencedirect.com/science/article/pii/ S0038092X04001525

[10] S. N. Alamri, The growth of CdTe thin film by close space sublimation 1 system physica status solidi (a) 200 (2) (2003) 352-360. doi:10.1002/ pssa.200306691. URL http://doi.wiley.com/10.1002/pssa.200306691

[11] H. M. Urbassek, Molecular-dynamics simulation of sputtering, Nuclear Inst. and Methods in Physics Research, B 122 (3) (1997) 427-441.

URL http://www.sciencedirect.com/science/article/pii/ S0168583X96006817

[12] A. NAKANO, A. Nakano, R. K. Kalia, R. K. KALIA, P. VASHISHTA, P. Vashishta, Growth of pore interfaces and roughness of fracture surfaces in porous silica: Million particle molecular-dynamics simulations, Physical Review Lettersdoi:10.1103/PhysRevLett.73.2336 URL http://journals.aps.org/prl/abstract/10.1103/PhysRevLett. 73.2336 
[13] W. Cheong, L. C. Zhang, Molecular dynamics simulation of phase transformations in silicon monocrystals due to nano-indentation, Nanotechnology 11 (3) (2000) 173-180.

URL http://iopscience.iop.org/0957-4484/11/3/307

[14] M. Yu, S. D. Kenny, The energetic impact of small CdxTey clusters on

घ Cadmium Telluride, Thin Solid Films 584 (C) (2015) 41-45. doi:10.1016/

$225 \quad$ j.tsf.2014.12.019

URL http://dx.doi.org/10.1016/j.tsf.2014.12.019

[15] M. Yu, S. D. Kenny, Using atomistic simulations to model cadmium telluride thin film growth, Journal of Physics: Condensed Matter (2016) 111doi:10.1088/0953-8984/28/10/105002 URL http://dx.doi .org/10.1088/0953-8984/28/10/105002

[16] L. Vernon, S. D. Kenny, R. Smith, E. Sanville, Growth mechanisms for TiO2 at its rutile (110) surface, Physical Review B 83 (7) (2011) 075412. doi:10.1103/PhysRevB.83.075412.

URL http://link.aps.org/doi/10.1103/PhysRevB.83.075412

235

[17] L. J. Vernon, R. Smith, S. D. Kenny, Modelling of deposition processes on the TiO2 rutile (110) surface, Nuclear Inst. and Methods in Physics Research, B 267 (18) (2009) 3022-3024. doi:10.1016/j.nimb.2009.06.093 URL http://www.sciencedirect.com/science/article/pii/ S0168583X09007101

240 [18] LAMMPS, LAMMPS Molecular Dynamics Simulator, http://lammps. sandia.gov, Accessd date (Feb. 2017).

URL http://lammps . sandia.gov

[19] S. Plimpton, Fast Parallel Algorithms for Short-Range Molecular Dynamics, Journal of Computational Physics.

245 URL http://www.sciencedirect.com/science/article/pii/ S002199918571039X 
[20] F. H. Stillinger, T. A. Weber, Computer simulation of local order in condensed phases of silicon, Physical Review B 31 (8) (1985) 5262-5271. doi:10.1103/PhysRevB.31.5262.

[21] X. W. Zhou, D. K. Ward, J. E. Martin, F. B. van Swol, J. L. Cruz-Campa, D. Zubia, Stillinger-Weber potential for the II-VI elements Zn-Cd-Hg-S-Se-

․ Te, Physical Review B 88 (8) (2013) 085309-14. doi:10.1103/PhysRevB. 88.085309 .

[22] J. M. Walls, Private Communication, Department of Electrical Engineering, Loughborough UniversityLeicestershire, U.K.

[23] H. J. C. Berendsen, J. P. M. Postma, W. F. van Gunsteren, A. DiNola, J. R. Haak, Molecular dynamics with coupling to an external bath, The Journal of Chemical Physics 81 (8) (1984) 3684. doi:10.1063/1.448118. URL http://link . aip.org/link/JCPSA6/v81/i8/p3684/s1\&Agg=doi

\section{Appendix B. List of Figures}




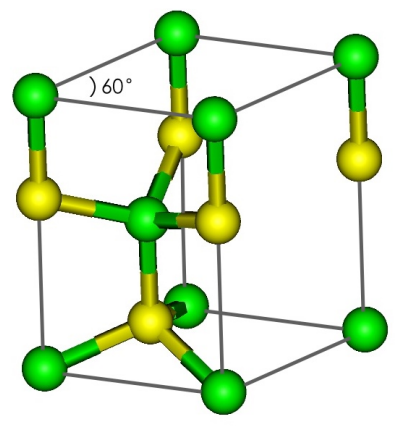

Figure B.1: The wurtzite structure of CdS, the green spheres represent the $\mathrm{Cd}$ and yellow ones the $\mathrm{S}$ atoms.

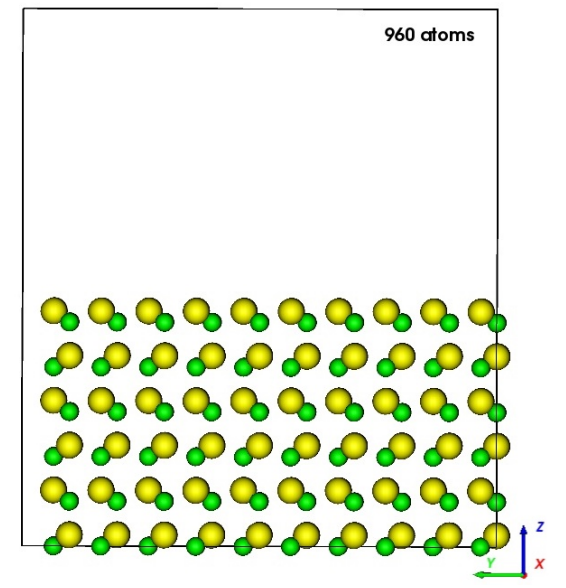

(a) Side view

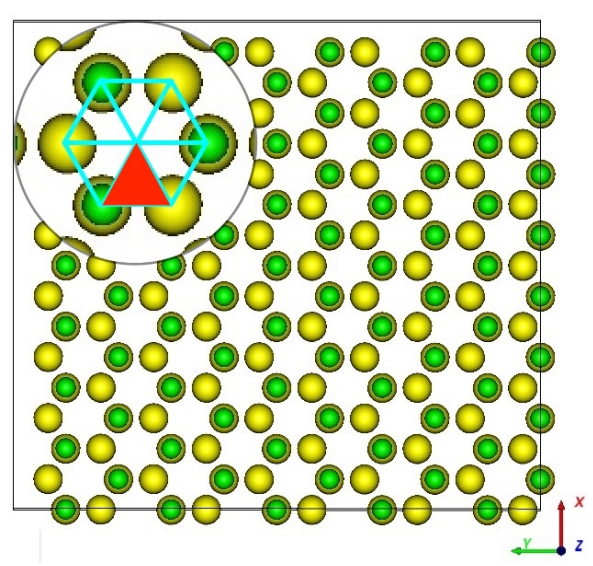

(b) Top view

Figure B.2: Side view and top view of a wurtzite (111) S-terminated CdS surface. Bigger yellow spheres represent $\mathrm{S}$ atoms, and smaller green spheres represent $\mathrm{Cd}$ atoms. The CdS lattice stacked in double-layers. Atoms in each double-layer are in a hexagonal shape. The red triangle areas in (b) indicate the smallest repeatable regions on the surface. 


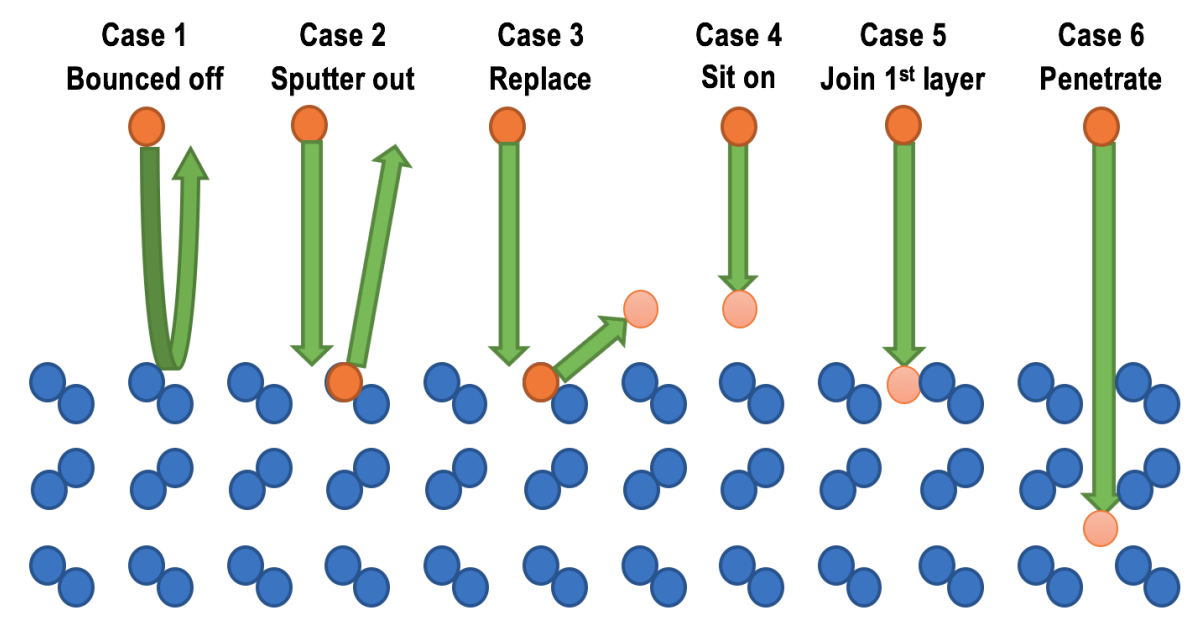

Figure B.3: The illustration graph for the 6 cases of the final states after impact as described in Table 1 


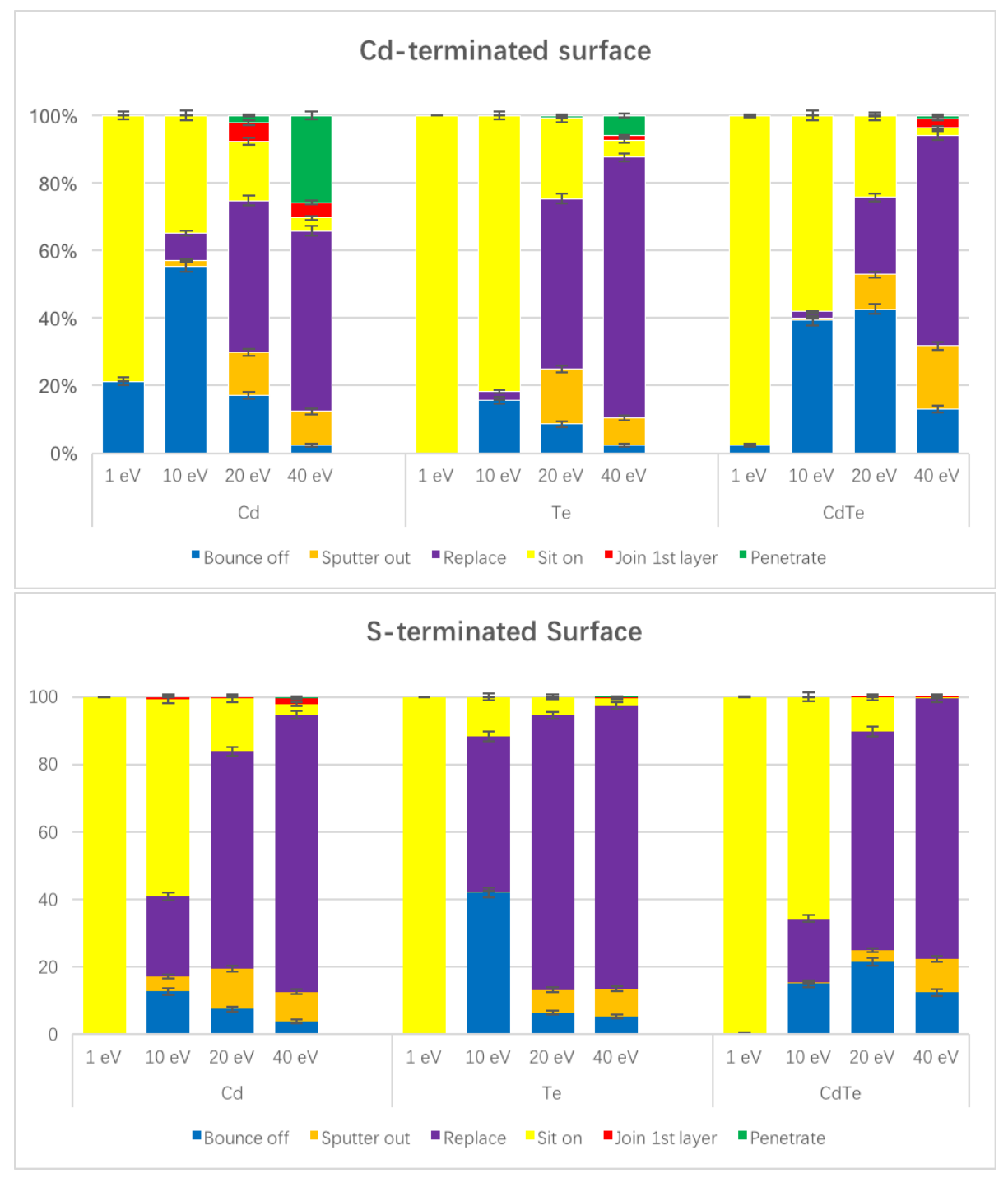

Figure B.4: Energetic impact results of small $\mathrm{Cd}_{x} \mathrm{Te}_{y} \quad(x, y=0,1)$ clusters on wurtzite (111) Cd-terminated and S-terminated CdS surfaces. The 6 cases of the final states are illustrated in Figure B.3 


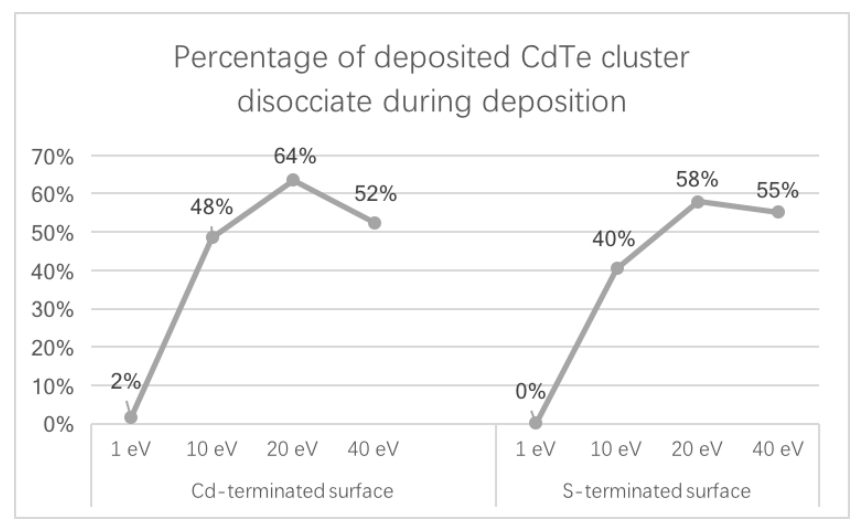

Figure B.5: Line graph for the percentage of deposited CdTe cluster dissociated during deposition.

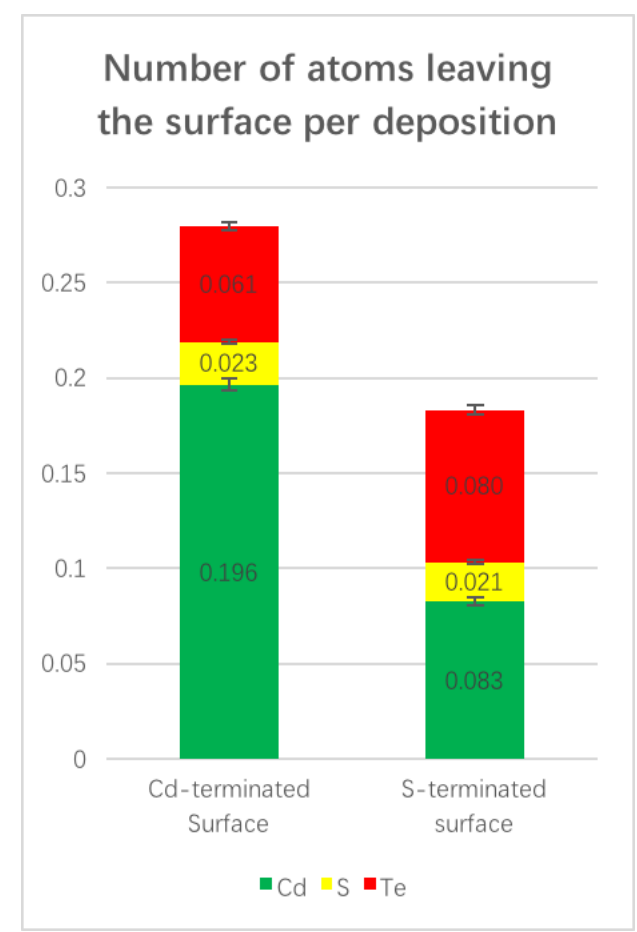

Figure B.6: Bar chart for the average number of atoms leave the surface per deposition. 